f. Phys. Chem. Solids Pergamon Press 1959. Vol. 12. pp. 28-40. Printed in Poland.

\title{
MAGNETIC PROPERTIES OF POLYCRYSTALLINE MATERIALS
}

\author{
D. M. GRIMES, R. D. HARRINGTON and A. L. RASMUSSEN \\ University of Michigan, Ann Arbor, Michigan \\ National Bureau of Standards, Boulder, Colorado
}

(Received 9 March 1959)

\begin{abstract}
The variation of the magnetic $Q$ with internal magnetization is discussed, using both the domain rotation and the domain-wall motion model of magnctization change. The variation of the reversible susceptibilities with magnetic moment is reported on four samples, and the results are compared with results from the frequency spectra in the initial and remanent states. The distribution of magnetic moments in the system as a function of the angle between individual and averaged moments is discussed in terms of an infinite series expansion in Legendre polynomials. The coefincients of the first four terms can be measured. Experimental data are given for the first three.
\end{abstract}

\section{INTRODUCTION}

THE magnetic susceptibility is defined as the ratio of resultant magnetization to magnetic field intensity. In ferrimagnets it is a function of both field strength and frequency. The reversible susceptibility is that susceptibility effective with a differential magnetic field. The differential field can be superimposed upon a finite biasing field. The reversible susceptibility depends upon the angle between biasing and differential fields, upon the magnetization level in the material, and upon the magnetic history of the specimen.

The susceptibility-producing mechanism is not completely understood. It is often assumed either that domain moments rotate in unison or that the domain walls move to change the

* Supported in part by the U.S. Air Force Office of Scientific Research and Development Command under Contract No. AF 18(603)-8. Reproduction in whole or in part is permitted for any purpose of the U.S. Government. net averaged moment of the sample. The reversible susceptibility and resulting differential magnetostriction for each mechanism were discussed and contrasted in an earlier paper, which will be referred to as $I .^{(1)}$

In that paper, the reversible quantities were discussed in terms of the distribution of magnetic moments. The calculations of predicted behavior of the reversible quantities with magnetic moment were carried out with the assumption that the moments would be distributed in some most probable state determined by a Boltzmann-type distribution of moments about the unit sphere. This argument has the merit that the direction of the moments is strongly influenced by crystallographic orientation, and that the orientation of neighboring grains can be considered independent. This does not mean, however, that the resulting moments must be randomly oriented. A factor of considerable interest, therefore, is a calculation of what the distribution of moments is in a magnetic material. 
The detailed calculation of $f(\Theta)$ is possible, at this time, in only the simplest cases. The basic difficulty is that the solution of this problem must involve the solution of essentially the same number of coupled differential equations as there are atomic moments. If the moments are treated in the aggregate, localized potential minima between neighboring grains and neighboring domains must be included, as must the ef fect of nonmagnetic inclusions. The dominant role played by this type of surface energy has been stressed by GoODENOUGH ${ }^{(2)}$.

The calculation of $f(\Theta)$, then, requires first the development of as realistic as possible a microscopic model of magnetic behavior followed with the distribution of this behavior over the sample. The microscopic models used in I were such that: (A) that the domain moments remained always oriented along the crystallographic directions which minimized theanisotrcpy energy, and (B) that the static moments remained along "easy" directions, but that the susceptibility arose by the rotation of the domain moments away from those directions in the dynamic case. Neither model is completely correct. Both are highly idealized and, for example, contain neither the "curling" concept discussed by BROWN ${ }^{(3)}$ and by FREI et al., (4) nor the effect of inhomogeneous fields. A more correct formulation awaits the inclusion of more accurate microscopic models.

There has, however, been ample precedent set for considering magnetic susceptibility as being due either to domain rotation or to domain-wall motion. This lamentable state is continued in the present paper.

It was assumied in I that the frequency of the measuring field was much less than the resonance or relaxation frequency of the magnet. Under these conditions, the magnetization-dependence of the transverse susceptibility on the biasing field is distinctly different for each of the assumed susceptibility sources as long as the anisotropy field remains much larger than the applied field.

The reversible susceptibility, as defined above, is reversible in the thermodynamic sense of zero energy dissipation for only the special case of zero applied frequency. An associated energy loss exists for all nonzero frequencies. This loss is describable as the imaginary part of the complex magnetic susceptibility, $(x=$ $\left.\chi^{\prime}-j \chi^{\prime \prime}\right)$. The magnetic $Q$ is defined to be the ratio of real to imaginary susceptibility. It is convenient in the following section to discuss the loss in terms of $Q$. The dependence of $Q$ upon magnetization, field directions, and source of susceptibility is briefly discussed in this paper.

Frequency spectra have commonly been used to investigate the susceptibility sources. This extensively used method depends upon the different dependence of the frequency of susceptibility fall-off on magnetization mechanism to distinguish between sources. ${ }^{(5-8)}$ Some investigators have also measured the spectra of the material magnetized to remanence and have utilized these results to aid spectral interpretation. ${ }^{(6-11)}$ EPSTEIN $^{(12)}$ and BIRKS ${ }^{(13)}$ in particular have utilized spectral information to obtain quantitative determinations of the amount of wall-motional and domain-rotational susceptibility.

\section{DISCUSSION}

\section{(a) Distribution function $f(\Theta)$}

The macroscopic material is considered to be composed of randomly oriented crystallites. The crystallites are sufficiently small so that the moment of each crystallite is much less than the aggregate moment of the sample. The crystalline orientation of each grain is taken to be independent of the orientation of its neighbors. For such a model the static moment is always parallel with the static field. 
The distribution $f(\Theta) \sin \Theta d \Theta$ is defined to be the fraction of the atomic moments in the system oriented at an angle between $\Theta$ and $\Theta+d \Theta$ with respect to the applied magnetic field. It is convenient to expand $f(\Theta)$ in an infinite series of Legendre functions such that:

$$
F(\Theta)=\sum_{n=0}^{\infty} A_{n} P_{n}(\cos \Theta)
$$

where $A_{n}$ is a function of the magnetic field present and the magnetic history of the specimen.

By definition,

$$
\left\langle\cos ^{n} \Theta\right\rangle=\frac{\int d \Omega \cos ^{n} \Theta f(\Theta)}{\int d \Omega f(\Theta)}
$$

where $\left\langle\cos ^{n} \Theta\right\rangle$ represents the average value of $\left\langle\cos ^{n} \Theta\right\rangle$ over the sample. Substituting equation (1) into equation (2) and integrating over the unit sphere yields:

$$
\left\langle\cos ^{n} \Theta\right\rangle=\frac{1}{2 A_{0}} \sum_{m=0}^{\infty} A_{m} \int_{-1}^{1} \gamma^{n} d \gamma P_{m}(\gamma) .
$$

Upon solving for the coefficients $A_{m}$ in equation (3), using the orthogonality of the Legendre functions,

$$
\frac{A_{m}}{A_{0}}=(2 m+1)\left\langle P_{m}(\cos \Theta)\right\rangle
$$

Thus the coefficient of the $m$ th term in the infinite series expansion for the distribution function can be determined if $\langle\cos \Theta\rangle,\left\langle\cos ^{2} \Theta\right\rangle$, $\ldots\left\langle\cos ^{m} \Theta\right\rangle$ are known. So, of course, the first coefficients are:

$$
\left.\begin{array}{l}
A_{1} / A_{0}=3\langle\cos \theta\rangle \\
A_{2} / A_{0}=5 / 2\left[3\left\langle\cos ^{2} \Theta\right\rangle-1\right] \\
A_{3} / A_{0}=7 / 2\left[5\left\langle\cos ^{3} \Theta\right\rangle-3\langle\cos \Theta\rangle\right] .
\end{array}\right\}
$$

Thus the distribution function can be experimentally determined if $\left\langle\cos ^{m} \Theta\right\rangle$ can be measured. The $A_{m}$ coefficients are functions of both magnetic field and magnetic history. The coefficient $A_{1} / A_{0}$ can be determined from the
$M-H$ loop. The coefficient $A_{2} / A_{0}$ can be determined from equation (5) and the magnetostriction $^{(14,15)}$ and independently from equations (9) and (10) of $I$, where it is stated that the susceptibility due to domain rotation is given by:

$$
\begin{aligned}
& \chi_{p}=\frac{3}{2} \chi_{0}\left(1-\left\langle\cos ^{2} \Theta\right\rangle\right) \\
& \chi_{t}=\frac{3}{4} \chi_{0}\left(1+\left\langle\cos ^{2} \Theta\right\rangle\right) .
\end{aligned}
$$

$A_{3} / A_{0}$ can be determined from a knowledge of $A_{1} / A_{0}$ and the use of equations (16) of $\mathrm{I}$, relating the differential magnetostrictions, namely:

$$
\left.\begin{array}{l}
d_{p}=\frac{3}{2} d_{0}\left(\langle\cos \Theta\rangle-\left\langle\cos ^{3} \Theta\right\rangle\right) \\
d_{t}=\frac{3}{4} d_{0}\left(\langle\cos \Theta\rangle+\left\langle\cos ^{3} \Theta\right\rangle\right)
\end{array}\right\}
$$

where $d_{0}=\frac{3 \lambda_{s} \chi_{0}}{M_{s}}$.

\section{(b) Magnetic $Q$ (Domain rotation)}

To estimate the magnetization-dependence of the magnetic $Q$ when the susceptibility arises from domain rotation, it is convenient to start with a single-crystal ferromagnet. The domain-rotation effects are assumed to obey the differential equation ${ }^{(16)}$

$$
\frac{\partial M}{\partial t}=\gamma(M \times H)-\frac{\alpha}{M_{s}} M \times \frac{\partial M}{\partial t},
$$

where $M$ is the magnetic moment, $\gamma$ is the magnetomechanical ratio, $M_{s}$ is the spontaneous moment, $H$ is the applied magnetic field, and $\alpha$ is a dimensionless parameter proportional to the power loss. The total sample moment is assumed to be the sum of moments from all crystallites. It is thus necessary to first calculate intergranular effects, then to sum up all moments over the polycrystalline sample. 
PARK $^{(8)}$ showed that if the magnetic moment of the grains neighboring a given grain averages that of the gross material, then grain size and particle interaction is described by a single constant $p$, defined by the equation

$$
H=h-p M
$$

where $\boldsymbol{H}$ is the effective differential field, $h$ is the applied differential field, and $M$ is the gross magnetization. The constant $p$ is a function of the localized packing and remains essentially independent of $M ; p$ can be regarded as the demagnetizing factor of the grain partially canceled by the moments of its neighbors.

Upon substituting equation (9) into equation (8) and using the additional assumptions that a large static biasing field is oriented in the $Z$-direction and sinusoidal time-dependence of an additional applied field, the reversible susceptibility matrix is given by: (equation (8) of I)

$$
Q^{r}=\frac{\omega_{0}}{\alpha \omega} \frac{1-\left(\frac{\omega}{\omega_{0}}\right)^{2}\left(1-\alpha^{2}\right)}{1+\left(\begin{array}{c}
\omega \\
\omega_{0}
\end{array}\right)^{2}\left(1-\alpha^{2}\right)},
$$

where $\omega$ is the applied radian frequency, and $\omega_{0}=\gamma H_{t} . H_{t}$, in turn, is the sum of the static applied and anisotropy fields, $H_{2 p}$ and $H_{a n}$, and the effective internal field $p M$.

I'hus:

$$
H_{t}=H_{a p}+H_{a n}+p M \text {. }
$$

In the low-frequency limit:

$$
Q^{r}=\frac{\omega_{0}}{\alpha \omega} .
$$

Note that $Q^{r}$ is independent of field direction. The low-frequency initial susceptibility is obtained by combining equations (5) and (10) of I. The result is:

$$
\chi_{0}=\frac{2 \omega_{1}}{3 \omega_{0}}
$$

$$
\chi=\frac{1}{4}\left(\begin{array}{cc}
{\left[1+\left\langle\cos ^{2} \Theta\right\rangle\right]\left(\chi_{-}+\chi_{+}\right),} & 2\langle\cos \Theta\rangle\left(\chi_{-}-\chi_{+}\right), 0 \\
-2\langle\cos \Theta\rangle\left(\chi_{-}-\chi_{+}\right), & {\left[1+\left\langle\cos ^{2} \Theta\right\rangle\right]\left(\chi_{-}+\chi_{+}\right), 0} \\
0, & 2\left[1-\left\langle\cos ^{2} \Theta\right\rangle\right]\left(\chi_{-}+\chi_{+}\right)
\end{array}\right),
$$

where \langle\rangle represents the average value in the polycrystal and $\Theta$ represents the angle between the spontaneous moment and the applied field. The susceptibilities $\chi_{ \pm}$are defined by the equation

$$
\chi_{ \pm}=\frac{M_{x} \pm i M_{y}}{H_{x} \pm i H_{y}}
$$

where $M_{x}$ and $H_{x}$ are the components of the differential magnetization and field in the $x$-direction and $i=\sqrt{-1}$. The $i$ operator represents a spatial rotation of $\pi / 2$ radians. The resultant algebra from combining equations (8), (9), and (11) for the $Q$ of the elements on the diagonal of equation (10), yields: where $\omega_{1}=\gamma M_{s}$. Combining equations (14) and (15),

$$
\chi_{0} Q^{r}=\frac{2 \omega_{1}}{3 \alpha \omega} .
$$

The product depends upon $\alpha$ and directly measurable quantities, and does not explicitly contain the biasing magnetic field through $\omega_{0}$.

\section{(c) Magnetic $Q$ (Wall motion)}

The change in magnetic moment due to $180^{\circ}$ wall movement is, in unit volume, given by:

$$
\Delta M=\sum_{k} 2 M_{s} A_{k} x_{k}
$$

where $A_{k}$ is the area of the $k$ th wall, and $x_{k}$ is 
the distance through which it is moved by an applied magnetic field $H$. The wall movement is presumed to be governed by the differential equation:

$$
2 \mu_{0} M_{s} H=f_{k} x_{k}+\beta \frac{d x \hat{k}}{d t}+m \frac{d^{2} x \hat{k}}{d t^{2}}
$$

where $f_{k}$ is the restoring constant for the $k$ th wall, $\beta$ depends upon the structure-insensitive properties of the material and the parameter $\alpha$ of equation (8), while $m$ depends only upon the structure-insensitive properties of the material. $\mu_{0}$ is the permeability of free space.

Combining equations (17) and (18), the wallmotional reversible susceptibility under sinusoidal excitation is found to be:

$\chi_{w}=4 \mu_{0} M_{s}^{2} \sum_{k} \frac{A_{k}}{f_{k}}\left[\frac{\left(1-\omega^{2} \frac{m}{f_{k}}\right)-j \omega \beta / f_{k}}{\left(1-\omega^{2} \frac{m}{f_{k}}\right)^{2}+\omega^{2} \frac{\beta^{2}}{f_{k}^{2}}}\right]$.

In the low-frequency limit, the real susceptibility and the $Q$ become:

$$
\begin{gathered}
\chi^{w}=4 \mu_{0} M_{s}^{2} \sum_{k}^{\top} \frac{A_{k}}{f_{k}} \\
Q^{w}=\frac{1}{\omega \rho} \frac{\sum A_{k} / f_{k}}{\sum A_{k} / f_{k}^{2}} .
\end{gathered}
$$

Thus for a given material at a fixed low frequency, the $Q$ varies only with $\beta, A_{k}$, and $f_{k}$.

The reversible susceptibility is given as a function of magnetization not only by the detailed mechanistic equation (20) above, but also by equations (2) and (18) of I. From these, if $\chi_{p}^{w}=\chi_{t}^{w}=\chi_{0}$ for virgin material with $M=0$ and if $\chi_{p}$ decreases montonically with increasing $M$, then so must $\chi_{t}$. Indeed the susceptibilities are related by the equation:

$$
\chi_{p}^{w}=\left[\frac{\chi_{t}^{w}}{1-\frac{d \ln \chi_{t}^{w}}{d \ln M}}\right] \text {. }
$$

If the average stiffness term $f_{k}$ increases with magnetization, then so will $Q$. Since both $A_{k}$ and $f_{k}$ vary with field orientation, the parallel and transverse $Q^{\prime}$ s will, in general, differ. This feature is distinctly different from domain rotation.

\section{(d) Initial and remanent susceptibility}

The initial susceptibility due to domain-wall motion has been approximated by BOZORTH ${ }^{(17)}$ for sinusoidal internal strains arising from magnetostrictive forces present when annealed material is cooled below the Curie temperature. The result is:

$$
\chi_{0}=\frac{4 \mu_{0} M_{s}^{2}}{3 \pi \lambda_{s}^{2} E}
$$

where $\lambda_{s}$ is the saturation magnetostriction and $E$ is the Young's modulus of the material. Likewise the initial susceptibility due to domain rotation can be approximated as:

$$
\chi_{0}=\frac{2 \mu_{0} M_{s}^{2}}{3 K_{1}}
$$

where $\mu_{0}$ is the permeability of free space and $K_{1}$ is the first-order anisotropy constant. From equations (23) and (24) it is apparent that in pure material the relative importance of the two susceptibility mechanisms depends upon the relative magnitudes of the effective anisotropy and magnetostrictive energy densities in the material.

For material with susceptibility arising by domain rotation, the relationship between the initial susceptibility and the resonant frequency should satisfy the equation:

$$
f_{0}=\frac{2 \gamma M_{s}}{2 \pi \cdot 3 x_{0}^{-}}
$$

where $\gamma$ is a constant taken to be $2.21 \times 10^{5}$ $\mathrm{m} / \mathrm{A}-\mathrm{sec}, f_{0}$ is the resonant frequency, $M_{s}$ is the spontaneous moment of the material, and $\chi_{0}$ is the initial susceptibility. 
BECKER and DöRING(18) discuss a model of magnetic remanence in which, as the field is decreased from the saturation value to zero, the moments rotate to occupy the same "easy" crystallographic directions occupied in virgin material, except that all components initially antiparallel to the saturation direction become parallel. For this model, the remanent magnetization is $0.5 M_{s}$ and the remanent rotational reversible susceptibilities equal the initial rotational susceptibility. This model is valid for hexagonal material with $K_{1}>0$. FoMENKC ${ }^{(9)}$ used it to interpret his permeability spectrum results. If this is also the position of maximum parallel and minimum transverse field susceptibility, then going around the $M-H$ loop the parallel susceptibility peak occurs for $\dot{M}$ decreasing in magnitude. The remanent and initial susceptibilities will be equal.

The rotational model of gross flux change in cubic crystals would predict moments oriented in the "easy" directions nearest the field directions at remanence. For this case the remanent and rotational susceptibilities taken from the results of I are given in Table 1 for all anisotropy coefficients save $K_{1}-0$. (These are the expected values from reference (1) for $\eta \rightarrow \infty$ ).

Table 1. Remanent results $M_{r} / M_{s}$

\begin{tabular}{c|c|c|c|c|c}
\hline & $\begin{array}{c}\text { Hexago- } \\
\text { nal } \\
K_{1}>0\end{array}$ & $\begin{array}{c}\text { Cubic } \\
K_{1}<\end{array}$ & $\begin{array}{c}\text { Cubic } \\
K_{1}>0\end{array}$ & $\begin{array}{c}\text { Hexago- } \\
\text { nal } \\
K_{1}<0\end{array}$ \\
\hline$M / M_{s}$ & 0.500 & 0.866 & 0.831 & 0.785 \\
$\chi_{p}^{r} / \chi_{0}^{r}$ & 1.000 & 0.366 & 0.449 & 0.500 \\
$\chi_{t}^{r} / \chi_{0}^{r}$ & 1.000 & 1.318 & 1.276 & 1.250 \\
\hline
\end{tabular}

An analysis of the relationships between remanent condition and the reversible susceptibility has been carried out by FREI and SHTRIKMAN (19) on the assumption that the reversible susceptibility is due to domain ro5 tation. Their analysis contains the assumption that the reversible quantities are due to rotation and that the expansion of magnetization in terms of applied field must obey the rotational equations through second order in the ratio of applied to anisotropy fields, as can be seen from their equation (10). Their resulting equation (29) can be put in the form:

$$
\frac{M_{r_{-}}}{M_{s}^{-}}=\frac{2 M_{s} \chi_{p}}{\left(\chi_{p}+2 \chi_{t}\right)^{2}}\left[\frac{d\left(\chi_{p}+2 \chi_{t}\right)}{d M}\right]
$$

for comparison with experimental values.

\section{EXPERIMENTAL}

\section{(a) Samples measured}

Four samples were subjected to detailed measurements and are reported here. The compositions of the samples are listed in Table 2. All except the magnesium ferrite sample were fabricated at the University of Michigan.

Table 2. Composition of ferrites surveyed

\begin{tabular}{l|l}
\hline \multicolumn{1}{|c|}{ Designation } & \multicolumn{1}{|c}{ Composition } \\
\hline F-1-2 & $\mathrm{Ni}_{0 \cdot 487} \mathrm{Zr}_{0 \cdot 533} \mathrm{Fe}_{2} \mathrm{O}_{4}$ \\
$\mathrm{~F}-6-2$ & $\mathrm{Ni}_{0 \cdot 168} \mathrm{ZV}_{0 \cdot 533} \mathrm{Co}_{0 \cdot 298} \mathrm{Fc}_{2} \mathrm{O}_{4}$ \\
AA-107-4 & $\mathrm{Fe}_{5} \mathrm{Y}_{3} \mathrm{O}_{12}$ \\
I-15-1 & $\mathrm{Mg} \mathrm{Fe}_{2} \mathrm{O}_{4}$ \\
\hline
\end{tabular}

The samples with designation starting F-1 and F-6 were prepared by first ball-milling the C.P. oxides weighed to the desired composition in acctone, then decanting, drying, and pressing into a toroidal pill. They were then heated rapidly to $1150^{\circ} \mathrm{C}$, heated slowly to $1375^{\circ} \mathrm{C}$ for $1 / 2 \mathrm{hr}$, slowly cooled to $1200^{\circ} \mathrm{C}$, and held for $2 \mathrm{hr}$. The furnace was then flushed with nitrogen and de-energized. The I-15-1 core was prepared by Dr. D. L. FrESH and is from the same material as that reported on previously by RADO et $a .^{(20)}$ as their type-F core. The AA-107-4 core was prepared by firing in air at $1350^{\circ} \mathrm{C}$ for $4 \mathrm{hr}$. The mixing 
and pressing procedure was the same as for the type-F-1 and F-6 cores.

\section{(b) Procedures and techniques}

All measurements were taken at ambient temperatures on toroidal samples, since this geometry avoids the complexities of demagnetizing factors. Coaxial line techniques were utilized for the spectral measurements. The complex susceptibility of the F-6 samples was measured from 1.0 to $40 \mathrm{Mc}$, using the radiofrequency permeameter in conjunction with a $Q$-meter. For the $F-1$ samples the real susceptibility from 0.1 to $40 \mathrm{Mc}$ and the imaginary susceptibility from 0.1 to $1.5 \mathrm{Mc}$ were measured with the permeameter. A fixedlength coaxial line and a radio-frequency bridge were used for the remaining higher loss measurements up to $50 \mathrm{Mc}$. From 50 to $5000 \mathrm{Mc}$, variable-length coaxial-cavity methods were used for complex susceptibility measurements on both types of samples.

The susceptibility and $Q$ for the variablefield measurements were taken with a $Q$-meter. For the transverse field measurements, the sample was placed between the pole faces of an electromagnet. The biasing field was along the axis of the toroid. Girdle windings about both outer and inner periphery of the toroid were used to determine the field and magnetization. An extra winding was placed about the toroid to furnish the biasing magnetic field when the parallel field measurements were taken.

(i) F-6 samples. Two different samples of F-6 material, code designated F-6-1 and F-6-17, were measured for the frequency spectra. The initial spectrum of F-6-1 is shown in Fig. 1. Both the initial and the remanent parallel spectra of F-6-17 are shown in Fig. 2. All spectra show but a single resonant peak in the real susceptibility. The resonant frequency as determined by the peak in the imag- inary susceptibility is seen to be about 130 Mc for F-6-1, with a corresponding low-frequency susceptibility of 47 . For F-6-17, the resonant frequency is about $75 \mathrm{Mc}$, with an initial low-frequency susceptibility of about 82. The remanent loss for F-6-17 also peaked at $80 \mathrm{Mc}$, with a low-frequency susceptibility of 75. An instability of a few per cent was noted in both samples between different measuring runs.

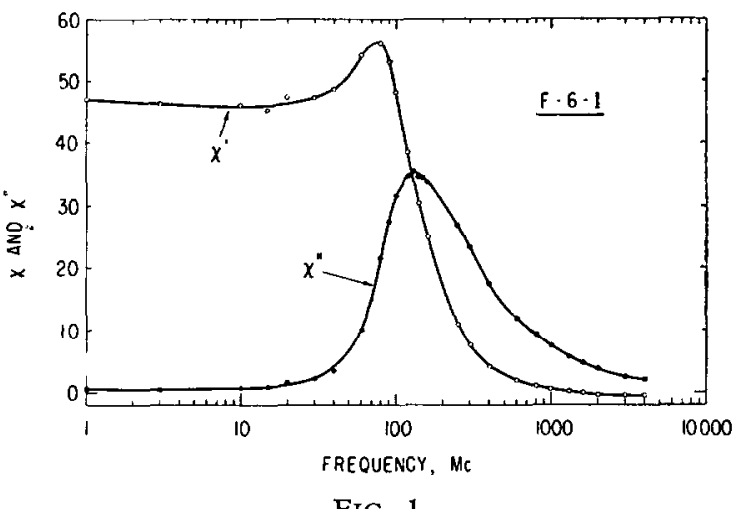

FIG. 1.

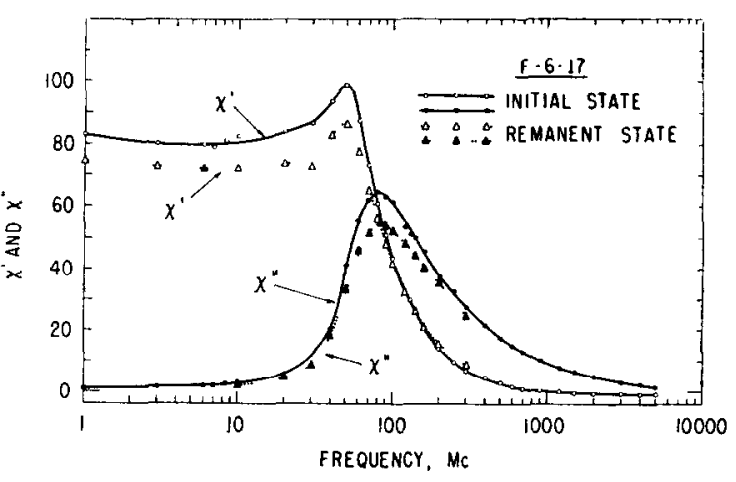

FIG. 2.

Comparing the initial and remanent curves, it is apparent that the positions of the peak in both real and imaginary susceptibilities occur at essentially the same frequency, and that the ratio of remanent to initial susceptibility is about 0.91 . 
The varation of the low-frequency susceptibility with magnetization is depicted in Fig. 3. These data were taken on sample F-6-2. The transverse susceptibility passes through a decided minimum near $M=0$. Both the measured $Q$ 's are essentially constant over a wide range of magnetization, and indeed were never observed to rise to a value equal to twice the initial value.

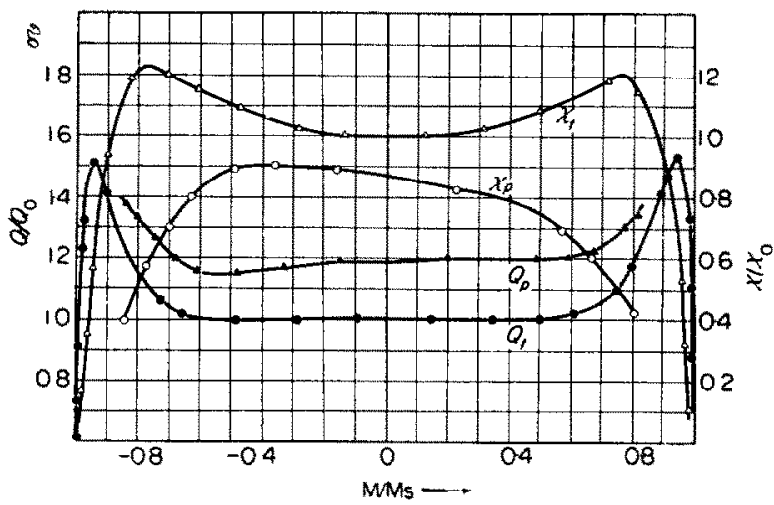

FIG. 3. Parallel and transverse field susceptibilities; Core F-6-2.

(ii) F-1 samples. The spectra of the sample code designated F-1-5 were tested. Fig. 4

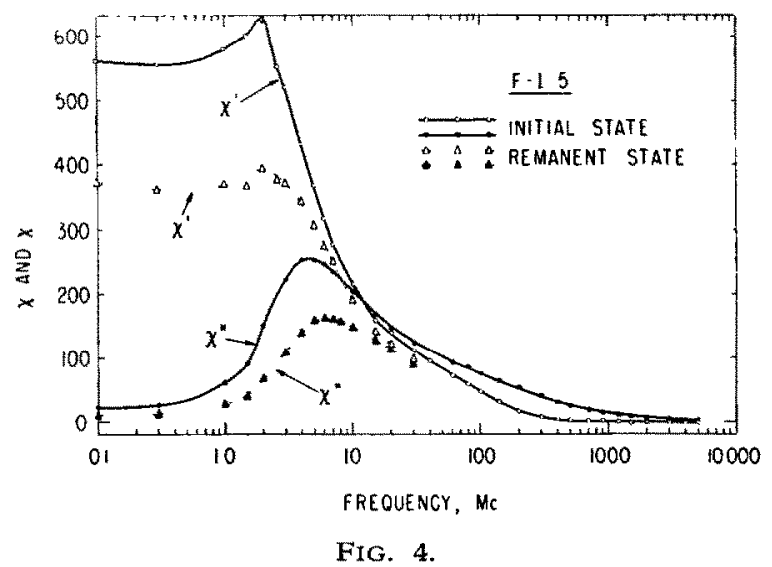

shows the complex susceptibility for both initial and parallel remanent states. The ini- tial spectrum of F-1-6 was also measured and found to be similar in all respects to F-1-5. The resonant frequency of F-1-5 was measured at $4.5 \mathrm{Mc}$ as determined by the peak in the imaginary value. The low-frequency susceptibility was measured as 561 . The ratio of remanent to initial value of susceptibility was 0.66 . The peak in imaginary susceptibility at remanence occurred at about $6 \mathrm{Mc}$.

The susceptibility was measured as a func-

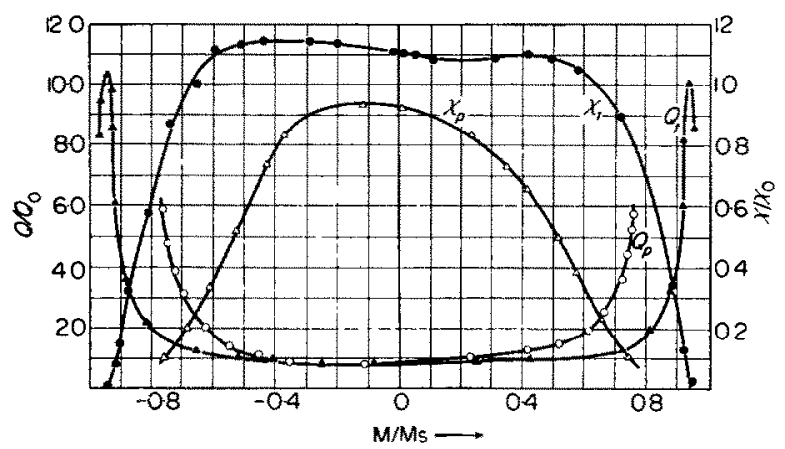

FIG. 5. Parallel and transverse field susceptibilities; Core F-1-2.

tion of the magnetization on specimen F-1-2 (see Fig. 5). For this core, the transverse susceptibility remained essentially constant over a wide range of magnetization values. The $Q$ 's pass through a minimum near $M=0$. As in the case of F-6-2, the transverse susceptibility remained always larger than the parallel value. The $Q$ 's increased rapidly with incrcasing ficld to a valuc more than 10 timcs the initial value.

(iii) $1-15-1$. The frequency spectrum for this material has been published by RADO et al..$^{(20)}$ and corresponds to their "Ferrite F". They conclude that the low-frequency susceptibility is predominately due to the movement of domain walls. The variation of the susceptibilities with magnetization is shown in Fig. 6. For this core, both the susceptibilities pass through a maximum in the vicinity of zero 
moment, while the $Q^{\prime}$ s pass through a minimum.

(iV) $A A-107-4$. The frequency spectra for this material have been published by two of

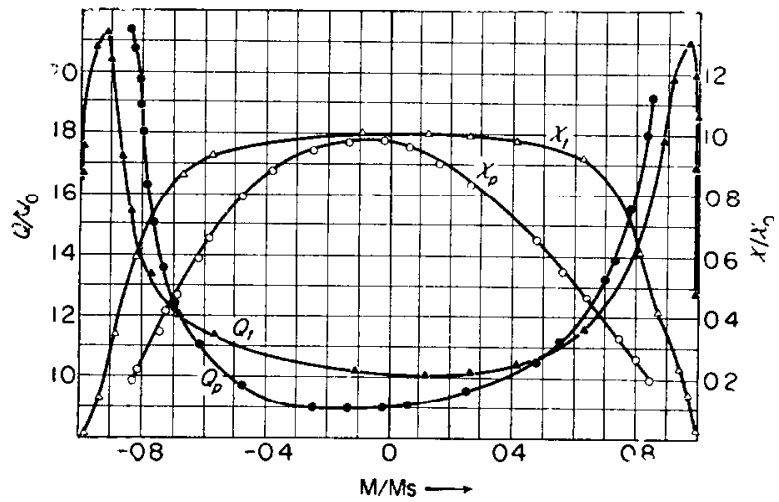

FIG. 6. Parallel and transverse field susceptibilities; Core I-15-1.

us. ${ }^{(21)}$ It was concluded on the basis of spectral information that the susceptibility was predominantely due to domain-wall motion. The variation of the susceptibilities with magnetization is shown in Fig. 7. The transverse susceptibility does not pass through a minimum.

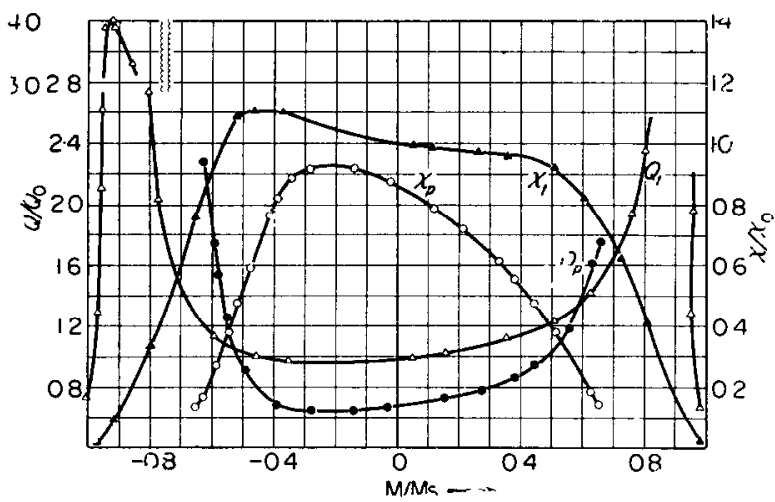

FIG. 7. Parallel and transverse field susceptibilities; Core AA-107-4.

\section{INTERPRETATION OF RESULTS}

The values of $f_{0}$ measured experimentally are listed in Table 3 , along with the values calculated from equation (25). The experimental value of $f_{0}$ was determined by the frequency of the peak value of imaginary susceptibility.

Table 3. Resonant frequency (Mc).

\begin{tabular}{l|c|c|c}
\hline Sample type & Measured & Calculated & $\frac{\chi_{p}(M=0)}{\chi_{p}(H=0)}$ \\
\hline F-6-1 & 130 & 179 & - \\
F-6-17 & 75 & 102 & 0.91 \\
F-1-2 & 45. & 15 & 0.66 \\
\hline
\end{tabular}

The expected variation of the parallel susceptibility with magnetization can be computed from the transverse susceptibility dependence. For the case of wall-motional susceptibility, equation (22) is the proper equation. For the case of rotational susceptibility, equation (10) of $I$ is the proper equation. For equation (10) to be useful, however, the variation of $\chi_{0}$ with applied field must be known. From equation (15) and the definition of $\omega_{0}$, it follows that in the absence of a detailed knowledge of the effective anisotropy field, which is always the case for polycrystalline material, $\chi_{0}$ is known only so long as the biasing field is small compared with the anisotropy field.

The magnetic parameters of the four cores are listed in Table 4.

Plots of $\chi_{p}$ computed from $\chi_{t}$ for each of the four samples measured are shown in Figs. 8-11 and compared with the experimentally measured curves. It is apparent that the rotational curve fits F-6-2 well and that the wall-motional curve fits I-15-1 well. The fit is not good for either of the other specimens, but nevertheless the wall-motional curve fits AA107 closer than the rotational curve. The conclusion that cores F-6-2 and I-15-1 have susceptibility arising predominantly from rotation and wall motion respectively is substan- 
Table 4. Magnetic parameters of four ferrimagnetic cores $(320 \mathrm{kc})$

\begin{tabular}{|c|c|c|c|c|c|c|c|c|c|c|}
\hline \multirow[b]{2}{*}{ Core } & \multicolumn{6}{|c|}{ Parallel } & \multicolumn{4}{|c|}{ Transverse } \\
\hline & $z_{0}{ }^{+}$ & $Q_{0}$ & $\chi_{p_{0}}$ & $Q_{p_{0}}$ & $\chi_{p r}$ & $Q_{p r}$ & $x_{t_{0}}$ & $Q_{t_{0}}$ & $y_{t r}$ & $Q_{t r}$ \\
\hline$-6-2$ & - & - & 46.5 & $72 \cdot 4$ & 39.6 & $71 \cdot 7$ & 52.8 & $60 \cdot 8$ & $62 \cdot 0$ & $61 \cdot 4$ \\
\hline$F-1-2^{\star}$ & 410 & $15 \cdot 2$ & 388 & $13 \cdot 2$ & 135 & $28 \cdot 1$ & 450 & $13 \cdot 5$ & 448 & 16.7 \\
\hline I-15-1 & - & - & $54 \cdot 2$ & 38.1 & $33 \cdot 3$ & 53.9 & $54 \cdot 2$ & 39.5 & $43 \cdot 6$ & 49.8 \\
\hline AA-107-4 & $36 \cdot 4$ & $10 \cdot 9$ & $31 \cdot 1$ & $7 \cdot 4$ & $30 \cdot 0$ & $8 \cdot 2$ & $36 \cdot 6$ & $10 \cdot 4$ & $40 \cdot 0$ & 9.7 \\
\hline
\end{tabular}

* Values at $500 \mathrm{kc}$.

+ The subscript ${ }_{0}$ indicates measurements in the virgin state, $p$ indicates parallel and $t$ transverse measurements. $p_{0}$ and $t_{0}$ represent measurements around the $M-H$ loop at the position $M=0$ in parallel and transverse fields. $p r$ and $t r$ represent similar measurements at the remanent position.

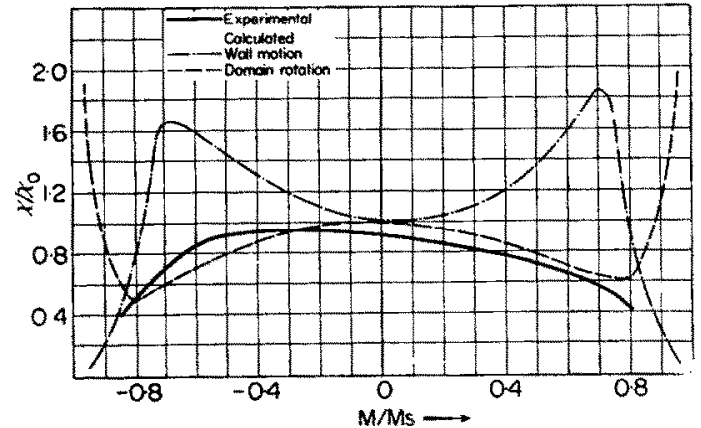

FiG. 8. Parallel reversible susceptibilities; Core F-6-2.

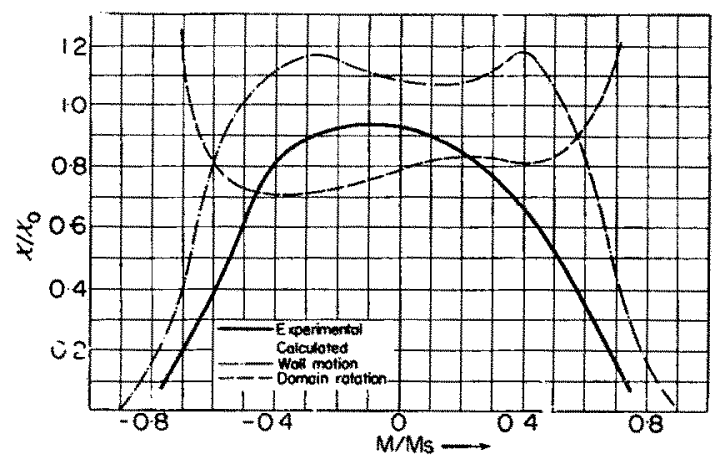

FIG. 9. Parallel reversible susceptibilities; Core F-1-2.

tiated by the spectral interpretation of Figs. 1 and 2 and by RADO et al..$^{(20)}$

The curve calculated for $\chi_{p}$ for rotation is valid so long as the anisotropy field remains much larger than the applied biasing field.

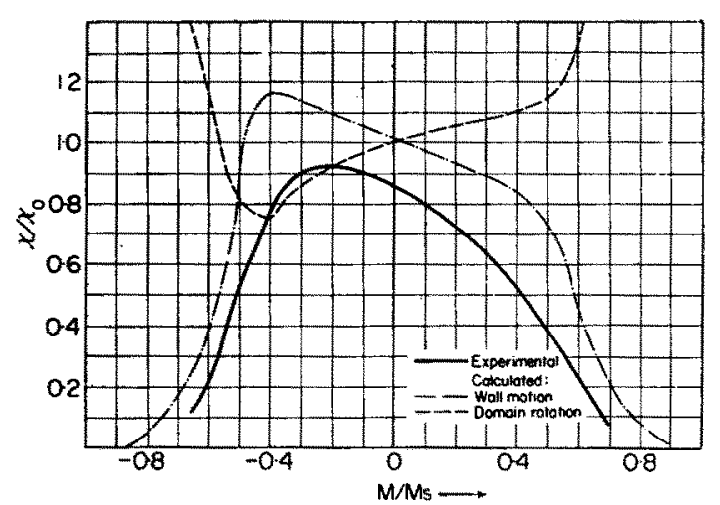

FIG. 10. Parallel reversible susceptibilities; Core AA$107-4$.

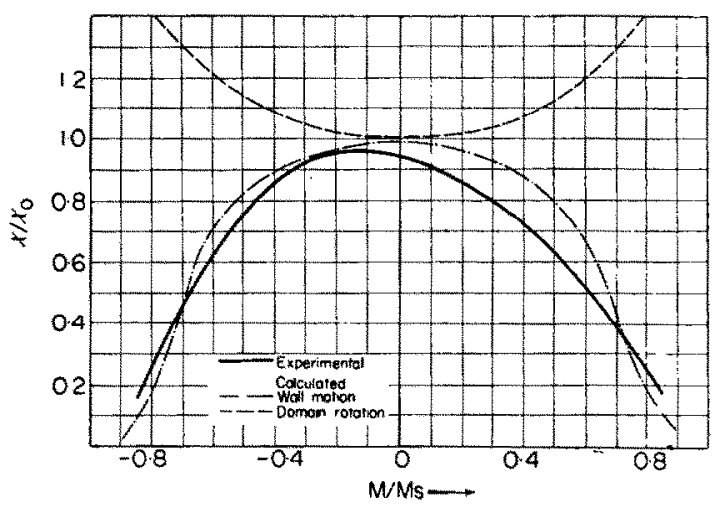

FIG. 11. Parallel reversible susceptibilities; Core I-15-1

F-6-2 contains cobalt, and therefore will have a large anisotropy field. Core F-1-2 s a mixed 
nickel-zinc ferrite with a corresponding small anisotropy. Thus the lack of agreement with rotational curves is not significant. For the anisotropy, but does satisfy the conditions of FREI and SHTRIKMAN. Table 5 compares the values of remanent moment calculated

Table 5

\begin{tabular}{l|c|c|c|c|c}
\multicolumn{1}{|c|}{ Specimen } & $\begin{array}{c}\text { Measured } \\
M_{r} / M_{s}\end{array}$ & $\begin{array}{c}\text { Calculated } \\
M_{r} / M_{s}\end{array}$ & $H_{c}$ & $\begin{array}{c}M_{s} \\
\times 10^{-5}\end{array}$ & $\begin{array}{c}M_{r} \\
\times 10^{-5}\end{array}$ \\
\hline AA-107-4 & 0.389 & 0.259 & 160 & 1.06 & 0.41 \\
F-6-2 & 0.660 & 0.041 & 127 & 3.58 & 2.37 \\
F-1-2 & 0.610 & 0.330 & 100 & 3.67 & 2.24 \\
I-15-1 & 0.730 & 0.675 & 175 & 1.35 & 0.98 \\
\hline
\end{tabular}

F-1-2 core, there was a slight but real minimum in the $\chi_{f}$ in the vinicity of zero moment. This feature is not consistent with wall motion and the accompanying $\chi_{p}$ behavior. Therefore it is concluded that domain rotation must be present. No such behavior was found in AA-107. In the spectral data, two peaks in the imaginary susceptibility were observed for AA-107, and only one for F-1-2. There must, therefore, be two important mechanisms for the AA-107. These mechanisms are assumed to be rotation and wall motion. For F-1-2, one can say only that domain-rotational effects enter. If wall-motional effects are present, the resonant frequency must occur at the same point as the rotational effect. The essential difference between F-1 and F-6 lies in the value of the anisotropy. Since rotational effects have been shown to exist in the high-anisotropy material, it is expected that they should also exist in the low-anisotropy material.

Material F-6-2 apparently fits the conditions imposed in this paper for the static conditions, namely oriented along easy crystallographic directions, and the susceptibility obeys the rotational equations. This material would not satisfy the conditions of FrEI and SHTRIKMAN ${ }^{(19)}$. On the other hand, F-1-2 does not satisfy the conditions as described in this paper, apparently because of the small from equation (26) with experimentally measured values. The agreement is not good, and indeed the best agreement is with the material whose susceptibility arises from wall motion.

Upon eliminating $\chi_{0}$ from equation (6), the average value of $\left\langle\cos ^{2} \theta\right\rangle$ is found to be:

$$
\left\langle\cos ^{2} \Theta\right\rangle=\left(\frac{2 \chi_{t}-\chi_{p}}{2 \chi_{t}+\chi_{p}}\right)
$$

Solving for $\left\langle\cos ^{3} \Theta\right\rangle$ from equations (7),

$$
\left\langle\cos ^{3} \theta\right\rangle=\left(\frac{2 d_{t}-d_{p}}{2 d_{t}+d_{p}}\right)\langle\cos \Theta\rangle .
$$

Note that $\left\langle\cos ^{2} \Theta\right\rangle$ should vary from $1 / 3$ in the demagnetized state to unity when the material is saturated. A similar wall-motional equation yields:

$$
\frac{2 \chi_{t}-\chi_{p}}{2 \chi_{t}+\chi_{p}}=\frac{1-2\left(\frac{d \ln \chi_{t}}{d \ln M}\right)}{3-2\left(\frac{d \ln }{d \ln M}\right)} .
$$

If the values of $\chi_{t}$ and $M$ are calculated assuming a Boltzmann distribution of moments, then $\chi_{t}=A M_{s} L(\eta) / \eta$ and $M=M_{s} L(\eta)$, where $A$ is a constant for the material, $L(\eta)$ represents the Langevin function of $\eta$, and $\eta=A H_{t}$, 
where $H_{t}$ is the totalized biasing field, including history, anisotropy, and applied field. Substituting these values into equation (29) yields a function which goes from $1 / 3$ for $M=$ 0 to unity for saturation.

Plots of $\frac{2 \chi_{t}-\chi_{p}}{2 \chi_{t}+\chi_{p}}$ as a function of $\langle\cos \Theta\rangle$ are shown in Figs. 12 and 13. In Fig. 12, the theoretical curve is the value of $\left\langle\cos ^{2} \Theta\right\rangle$ calculated for domain rotation. In Fig. 13, the theoretical

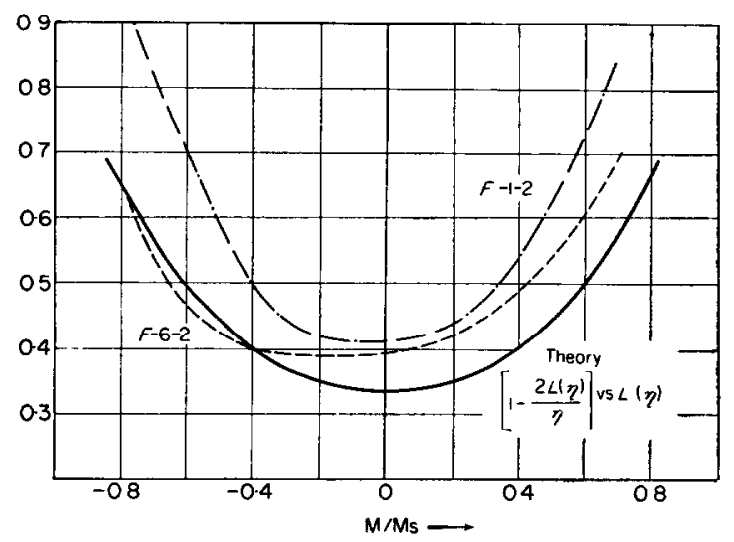

FIG. 12. Variation of $\left\langle\cos ^{2} \Theta\right\rangle$ with $\langle\cos \Theta\rangle$.

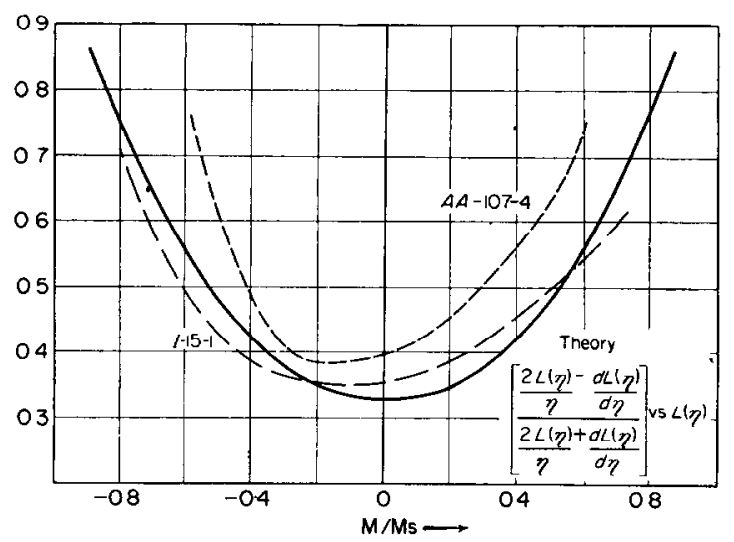

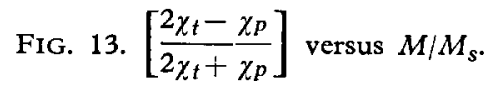

curve is based upon domain-wall motion. Both curves are based upon Boltzmann distribution of moments. The rotational curve attributed to domain rotation can be interpreted in terms of variation from the most probable condition. For F-6-2, as the moment is decreased, moments parallel and antiparallel are initially in excess of the most probable condition. However, as $M$ is further decreased towards zero, the parallel-antiparallel components increase to larger than the most probable value. This can be considered the reason for the transverse susceptibility at zero moments being larger than the initial susceptibility, and the corresponding parallel susceptibility at zero moment being smaller than either. Sample F-1-2, if the susceptibility is assumed entirely rotation, maintains at all times a predominant parallel-antiparallel moment configuration. The value of $A_{2}$ from equation (5) as a function of magnetic moment can be read directly from Fig. 12 for sample F-6-2.

More detailed determinations and analyses of the distribution functions in other materials must await simultaneous parallel and transverse susceptibility and differential magnetostriction measurements.

\section{CONCLUSIONS}

The magnetic properties of a ferromagnetic system can be described in terms of a distribution of magnetic moments about the unit sphere. This function cannot be calculated in detail for macroscopic systems. However, assuming idealized models of magnetic behavior such as magnetization by domain-wall motion or magnetization by domain rotation, some reversible properties of ferromagnets can be compared without detailed knowledge of the distribution function. If a detailed distribution function is assumed, then, of course, the magnetic properties can be calculated in detail.

The inverse of this calculation can also be made. The procedure consists of firstly expanding the distribution function in an infinite ser- 
ies of Legendre polynomials. Due to the orthogonality properties of these functions, the coefficients of each term in the infinite series can be evaluated if the weighted average value of the proper power of $\langle\cos \theta\rangle$ over the sample is known; $\langle\cos \Theta\rangle$ itself is, of course, proportional to the magnetic moment of the sample. Under certain conditions $\left\langle\cos ^{2} \Theta\right\rangle$ is proportional to the static magnetostriction. It can also be found from a knowledge of the two reversible susceptibilities, if domain rotation is the source of the susceptibility. Further, under the same susceptibility conditions, the differential magnetcstriction can be utilized to find $\left\langle\cos ^{3} \Theta\right\rangle$ and one additional coefficient in the expansion.

The combination of frequency spectra and magnetization dependence of the susceptibilities on the same sample types is utilized to examine the source of the susceptibility. In agreement with other investigators, it is found that the major susceptibility source depends upon the type of ferrite, as well as the method of preparation. The nickel-zinc-cobalt ferrite F-6-2 shows distinctive domain-rotational effects. Sample I-15-1 shows wall-motional effect.

Acknowledgements-The authors wish to acknowledge the invaluable assistance of $\mathrm{Mr}$. R. M. OlsoN, Mr. A. H. VOELKER, and Mrs. P. A. MARChELlo.

\section{REFERENCES}

1. Grimes D. M., f. Phys. Chem. Solids 3, 141 (1957).

2. Goodenough J. B., Phys. Rev. 95, 917 (1954).

3. BROWN W. F., Jr., Phys. Rev. 105, 1479 (1957).

4. Frei E. H., Shtrikman S. and Treves D., Phys. Rev. 106, 446 (1957).

5. SNOEK J. L., Physica 14, 207 (1948).

6. Wijn H. P. J., Gevers M. and Van Der Burgt C. M., Rev. Mod. Phys. 25, 91 (1953).

7. Brown F. and Gravel C. L., Phys. Rev. 98, 442 (1955).

8. PARK D., Phy's. Rev. 97, 60 (1955).

9. Fomenko L. A., Sov. Phys. JETP 3, 19 (1956).

10. Harrison S. E., KRIESSMaN C. J. and Pollack S. R., Phys. Rev. 110, 844 (1958).

11. RADO G. T., WRIGHT R. W. and EMERSON W. H., Phys. Rev. 80, 273 (1950).

12. Epstein D. J., Proc. Boston Conf. Amer. Inst. Elect. Engrs. 493, T-91 (1957).

13. Birks J. B., Proc. Inst. Elect. Engrs. Part B, Suppl. No. 5, 179 (1956).

14. Brown W. F., Jr., Phys. Rev. 53, 482 (1938).

15. LeE. E. W., Proc. Phys. Soc. 72, 249 (1958).

16. Gilbert T. L., The Phenomenological Theory of Ferromagnetism. Armour Research Foundation (1 May, 1956).

17. BOzORTH R. M., Ferromagnetism p. 822. Van Nostrand, New York (1951).

18. BECKER R. and DóRING W., Ferromagnetismus Edward Bros., Ann Arbor (1943).

19. Frei E. H. and Shtrikman S., Proc. Boston Conf. Amer. Inst. Elect. Engrs. 502, T-91 (1957).

20. RADO G. T., Folen V. J. and EMERSON W. H., Proc. Inst. Elect. Engrs. Part B, Suppl. No. 5, 198 (1956).

21. Harrington R. D. and Rasmussen A. L., Proc. Inst. Radio Engrs. 47, 98 (1959). 